\title{
Membrane cholesterol interferes with tyrosine phosphorylation but facilitates the clustering and signal transduction of EGFR
}

\section{Authors}

Michio Hiroshima ${ }^{1,2, *}$, Mitsuhiro Abe ${ }^{2}$, Nario Tomishige ${ }^{3}$, Françoise Hullin-Matsuda ${ }^{4}$, Asami Makino $^{2}$, Masahiro Ueda ${ }^{1,5}$, Toshihide Kobayashi ${ }^{3}$, and Yasushi Sako ${ }^{2}$

\section{Affiliations}

${ }^{1}$ Laboratory for Cell Signaling Dynamics, RIKEN BDR, 6-2-3, Furuedai, Suita, Osaka, 565-0874, Japan.

${ }^{2}$ Cellular Informatics Laboratory, RIKEN CPR, 2-1 Hirosawa, Wako 351-198, Japan.

${ }^{3}$ Laboratory of Bioimaging and Pathologies, University of Strasbourg, 74 route du Rhin, CS 60024-67401 Illkirch, France

${ }^{4}$ CarMeN Laboratory, University of Lyon, Inserm U1060, INRAe U1397, Université Claude Bernard Lyon 1 Pierre-Bénite, France

${ }^{5}$ Laboratory of Single Molecule Biology, Graduate School of Frontier Biosciences, Osaka University, 1-3 Yamadaoka, Suita, Osaka 565-0871, Japan.

\section{* Corresponding Author}

Correspondence should be addressed to Michio Hiroshima (m_hiroshima@riken.jp). 


\section{Summary}

Epidermal growth factor receptor (EGFR) activates major cell signaling pathways that regulate various cell responses. Its dimerization and clustering coupled with its lateral mobility are critical for EGFR function, but the contribution of the plasma membrane environment to EGFR function is unknown. Here we show, using single-molecule analysis, that EGFR mobility and clustering are altered by the depletion of cholesterol or sphingomyelin, major lipids of membrane subdomains, causing significant changes in EGFR signaling. When cholesterol was depleted, the subdomain boundary in EGFR diffusion disappeared, the fraction of EGFR pre-dimers was increased, and the ligandinduced phosphorylation of EGFR was enhanced. In addition, the depletion of either lipid prevented the formation of immobile clusters after EGF association and decreased the phosphorylation of downstream proteins. Our results revealed that cholesterol plays dichotomous roles in the signaling pathway of EGFR and that clustering in the membrane subdomains is critical for EGFR signal transduction. 


\section{Introduction}

2 Epidermal growth factor receptor (EGFR), a receptor tyrosine kinase, is a major

3 regulator of several intracellular signaling cascades by receiving extracellular ligands at

4 the plasma membrane. EGFR signaling transfers information to the well-known RAS-

5 MAPK pathway, inducing essential cell responses such as proliferation, differentiation,

6 migration, apoptosis, and others. Defects in EGFR function affect cellular responses,

7 often inducing hyperactivated signaling to causes carcinoma and other diseases

8 (Carpenter et al., 1978; Lemmon and Schlessinger, 2010). Ligand-bound EGFR is auto-

9 phosphorylated when EGFR takes a dimer structure to activate downstream signaling.

10 Even before the ligand binding, dimer formation (pre-dimer) occurs (Hiroshima et al.,

11 2012, 2018; Martin-Fernandez et al., 2002; Teramura et al., 2006; Yu et al., 2002), but

12 usually the dimer cannot evoke whole cell activation and instead results in only higher

13 ligand-affinity than the monomer. The ligand binding alters the pre-dimer state to an

14 active dimer state capable of auto-phosphorylation. Previous studies have also indicated

15 the existence of receptor pre-clusters larger than the dimer (Tao and Maruyama, 2008;

16 Webb et al., 2008). Since the dimerization and cluster formation are driven by a

17 molecular collision during diffusion along the plasma membrane, the membrane

18 environment is reflected in the EGFR behavior (Arkhipov et al., 2013; Lin et al., 2016;

19 Valley et al., 2015) and affects the EGFR signaling (Lajoie et al., 2007). To elucidate

20 the membrane environment effects, observation of the spatiotemporal behaviors of

21 individual EGFR molecules in cells is necessary.

Single-molecule trajectory analysis combined with the hidden Markov model

23 (HMM) based on machine learning methods (Okamoto and Sako, 2012; Rabiner, 1989)

24 has been used to infer molecular state transitions along the trajectory of an individual

25 molecule (Chung et al., 2010; Low-Nam et al., 2011; Persson et al., 2013). EGFR was

26 shown to transit between three motional states; namely, immobile, slow-, and fast- 
27 mobile, which were determined in terms of the size of the diffusion coefficient

28 (Hiroshima et al., 2018; Yasui et al., 2018). We previously found that EGFR molecules

29 in the slow-mobile state showed a confined diffusion surrounding the trajectory of the

30 immobile state in which the position of the molecules fluctuated within a confined area

$31(\sim 60 \mathrm{~nm})$ only two times larger than the localization accuracy. Fast-mobile EGFR

32 molecules moved in space between the slow-mobile compartment with simple diffusion.

33 The clustering states, which correspond to the number of EGFR molecules moving

34 together, were determined from the brightness of the GFP fluorescence probing EGFR.

35 After EGF stimulation, the EGFR clustering state shifted initially from monomer to

36 dimer and subsequently to larger clusters concurrent with a shift to a slower mobility state. Immobile clusters are the primary interaction sites with the downstream protein GRB2. The dissociation kinetics between EGFR and GRB2 is specifically slower in immobile clusters, suggesting they play a significant role in the signal transduction in cells (Hiroshima et al., 2018). Overall, these results suggested that lateral mobility, clustering, and signal activation are closely correlated in EGFR.

The confinement size of the slow-mobile diffusion we have observed $(\sim 200 \mathrm{~nm})$

43 is equivalent to the size often reported for lipid rafts (a membrane subdomain), which are a liquid-ordered phase segregated from the bulk region (liquid disordered phase) of the plasma membrane (Semrau and Schmidt, 2009). However, little is understood about

46 the relationship between EGFR behavior and the membrane environment. Cholesterol and sphingomyelin are well-known major components of lipid rafts and can be depleted from the plasma membrane by treatment with methyl- $\beta$-cyclodextrin $(\mathrm{M} \beta \mathrm{CD})$, which extracts cholesterol from the membrane to its hydrophobic cavity (Zidovetzki and Levitan, 2007), or sphingomyelinase (SMase), which catalyzes the breakdown of

51 sphingomyelin to phosphorylcholine and ceramide; ceramide is then converted to sphingosine and sphingosine-1-phosphate (S1P) and transported out of the cells 
54 have been reported to disperse and affect the physical properties of lipid rafts,

55 respectively (Cremesti et al., 2002; Smith et al., 2010), indicating they alter EGFR

56 behavior. These perturbations offer information on how cellular signaling is affected by

57 the plasma membrane environment through the dynamics of EGFR behavior at the

58 molecular level.

To understand the dependency of EGFR behavior, including the localization,

60 mobility, clustering, and their coupling, on the membrane structure, the present study

61 employed single-molecule analysis while depleting cholesterol or sphingomyelin.

62 Furthermore, assessments of the receptor and its downstream activity were carried out

63 to reveal the correlation between EGFR behavior and cellular signaling. 


\section{Results}

\section{Cholesterol- but not sphingomyelin-depletion enhanced EGF-induced EGFR 66 phosphorylation}

67 CHO-K1 cells were transfected with EGFR-GFP and treated with M $\beta \mathrm{CD}$ or

68 sphingomyelinase for the lipid depletion. Cholesterol was reduced to $33 \%$ and $16 \%$ with

695 and $10 \mathrm{mM} \mathrm{M} \beta C D$ treatment, respectively, according to GC-FID or GC-MS

70 measurements (Fig. 1a). Similar depletion was also confirmed by the exogenous

71 addition of fluorescent EGFP-labeled $\theta$ toxin, a probe of free cholesterol (Fig. 1b), that

72 binds to the cells. Sphingomyelin was reduced to $18 \%$ by sphingomyelinase treatment

73 (Fig. 1b) based on the fluorescence of GFP-labeled lysenin, a specific probe of

74 sphingomyelin (Fig. 1b). The observed fluorescence of $\theta$ toxin-GFP in

75 sphingomyelinase-treated cells and lysenin-GFP in M $\beta C D$-treated cells were the same

76 as in non-treated cells (Fig. S1), indicating that the specific depletion of one lipid

77 neither affected the content of the other lipid in cells.

The time-course of EGFR phosphorylated at Y1173 in cholesterol-depleted and control cells reached a maximum at 1-3 minutes after EGF stimulation according to Western blotting results (Fig. 1c and S2a). The phosphorylation level under cholesteroldepletion was higher than that in the control condition and was dependent on the M $\beta C D$ concentration. The phosphorylation of EGFR at Y1173 and Y1068 was increased by the cholesterol depletion two minutes after the stimulation (Fig. 1d). The half-maximal effective concentrations $\left(\mathrm{EC}_{50}\right)$ of EGF was almost the same between control and cholesterol-depleted conditions: $1.9 \mathrm{nM}$ and $2.1 \mathrm{nM}$ for pY1173, and 1.5 $\mathrm{nM}$ and 1.3 nM for pY1068, respectively. Hill coefficients indicating no cooperativity (0.6-1.0) were not changed by the depletion. After 30 nM EGF stimulation, the cholesterol- 
89 Y1173, respectively, compared with the control condition (Fig.1e and S2b).

90 Sphingomyelin depletion did not affect the phosphorylation of Y1068 or Y1173

91 significantly (Fig. 1e).

$92 \quad$ Fig. 1
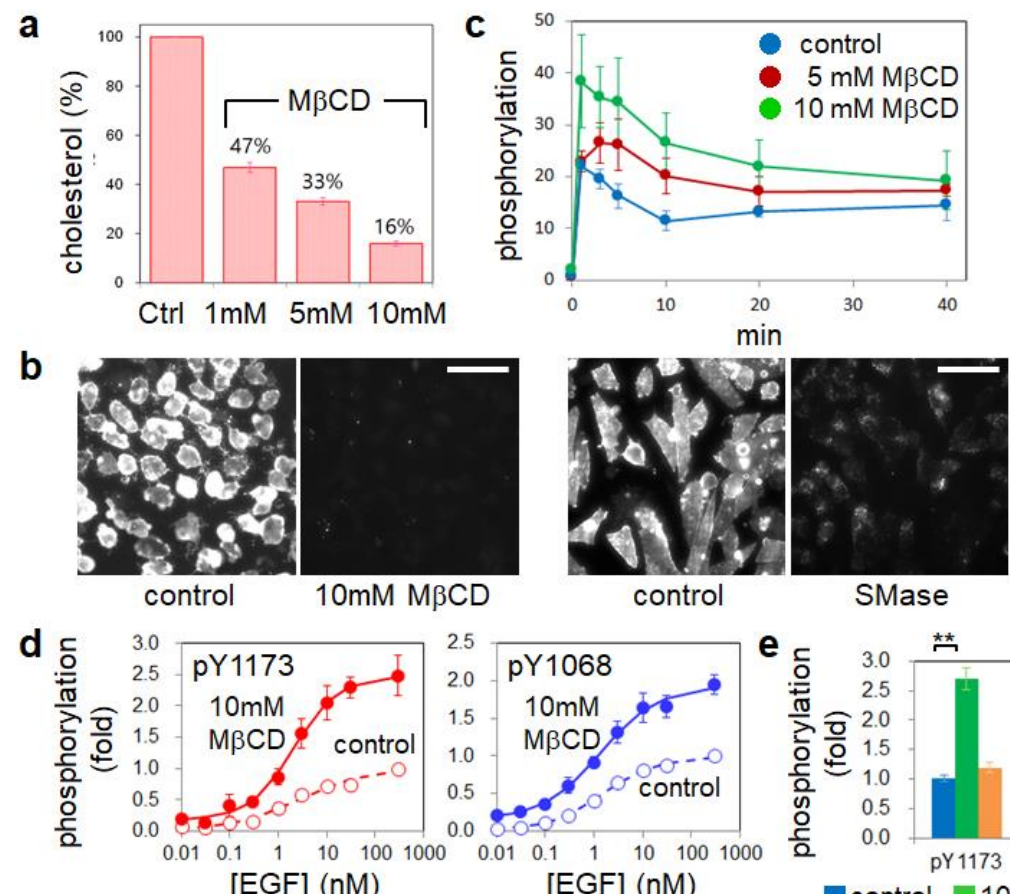

[EGF] (nM)
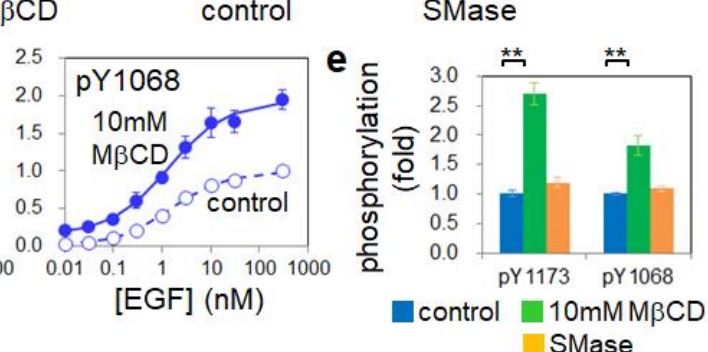

93 Lipid-depletion and EGFR phosphorylation. a. Cholesterol content in M $\beta C D$-treated

cells. The amount of cholesterol was normalized to that in non-treated cells. b.

95 Fluorescence images of wild-type CHO-K1 cells labeled with GFP-conjugated lipid probes. Left: $\theta$ toxin-labeled cells. Right: Lysenin-labeled cells. Scale bars, $50 \mu \mathrm{m}$. c.

97 Time-course of the EGF-induced EGFR phosphorylation (pY1173) ( $\mathrm{n}=3$ trials). Fold-

98 changes relative to phosphorylation at 0 min are indicated. d. Dose-response curves for

99 EGF-induced tyrosine phosphorylation in EGFR. e. Comparison between the

100 phosphorylation levels at 2 min after $30 \mathrm{nM}$ EGF stimulation. $* * \mathrm{p}<0.01$ (t-test). a-e,

101 Error bars: SE. All data points are shown in Fig. S2. 


\section{Cholesterol-depletion enlarged the slow-mobile region before EGF stimulation}

104 To understand how the differences in phosphorylation arose among the control and

105 cholesterol-depleted conditions with consideration of EGFR behavior, we applied

106 single-molecule imaging of EGFR-EGFP on the plasma membrane of living cells. We

107 analyzed the trajectories of individual fluorescent spots using an HMM-based machine

108 learning method to assign every step along the trajectories with specific motional and

109 clustering states. The movements consisted of immobile, slow-, and fast-mobile states in

110 all lipid conditions (Fig. S3 and Table S1). The depletion of either lipid increased the

111 diffusion coefficient in the immobile state and the fraction of the slow-mobile state

112 while decreasing the fraction of the fast-mobile state. Sphingomyelin depletion also

113 increased the diffusion coefficient of the fast-mobile state. The observed changes in the

114 diffusion coefficients were consistent with previous reports indicating that membrane

115 fluidity is reduced by the addition of cholesterol (Tabas, 2002) or sphingomyelin

116 (Makdissy et al., 2015). Trajectories of the lateral motion (Fig. 2) showed that

117 cholesterol-depletion enlarged the diffusion region especially during the slow-mobile

118 state (Fig. 2a, orange) but that the sphingomyelin-depletion had little effect. These

119 trajectories reflect the properties of the time evolution of the mean square displacement

120 (MSD; Fig. 2b and S4).

121 MSD profiles of confined and simple diffusion were fitted with the following

122 equations, respectively,

$$
M S D=L^{2} / 3\left\{1-\exp \left(-12 \frac{D_{1} t}{L^{2}}\right)\right\},
$$

$$
M S D=4 D_{2} t
$$


125 where $D_{1}$ and $D_{2}$ are the diffusion coefficients, $L$ is the confinement length, and $t$ is the

126 diffusion time. The suitable diffusion mode for each MSD profile was determined using

127 Akaike's information criterion (AIC), which was calculated using the equation below,

$$
A I C=\log \left(\frac{R S S}{N}\right)+2 k / N
$$

129 where $R S S$ is the residual sum of squares between the data and the model, $N$ is the

130 number of data points, and $k$ is the number of parameters. The model with the higher

131 AIC was selected. In the control condition, confined diffusion was observed in the

132 immobile and slow-mobile states, but the fast-mobile state showed simple diffusion.

133 The confinement lengths $(L)$ of the immobile state were $60 \mathrm{~nm}$ for all cluster sizes

134 (monomer, dimer, and higher-order clusters). $L$ for the slow-mobile state was equivalent

135 to the size of well-known membrane subdomains (including lipid rafts). Finally, the

136 mobility of the monomer $(\sim 310 \mathrm{~nm})$ was more confined than the mobility of the other

137 clusters $(\sim 570 \mathrm{~nm})$.

When cholesterol was depleted, the diffusion mode in the slow-mobile state was

139 altered from confined to simple diffusion for all cluster sizes. The cholesterol depletion

140 had little effect on the MSD of both the immobile and fast-mobile states or on the

141 distance between the centers of the immobile state regions (Fig. S5). When

142 sphingomyelin was depleted, the slow mobile EGFR still showed confined diffusion,

143 but the confinement was less in comparison with the control condition $(\sim 720 \mathrm{~nm}$ for $\geq$

144 dimer). In Fig. 2c, individual trajectories were superimposed to exhibit the expanding

145 diffusion area of EGFR, for which the center of the first immobile state in each

146 trajectory was shifted to the origin. The second and later immobile states are seen as

147 small islands separated from the first region, and the slow- and fast-mobile states were

148 distributed around the immobile states (Fig. 2c, left). Typical trajectories (Fig. 2c, right)

149 rarely showed a direct transition between the immobile and fast-mobile states, as 
150 observed in the transition probability (Table S1). Transitions between the immobile and

151 slow-mobile states often occurred at the surrounding boundary of the region for an

152 immobile state, suggesting that an EGFR particle in the immobile state was trapped

153 within a $60-\mathrm{nm}$ membrane subdomain that was stable during the observation time.

154 Boundaries between the slow-mobile and fast-mobile regions were obscure. Thus, the

155 small core area surrounded by a region several hundred nanometers wide forms a

156 membrane subdomain in which EGFR molecules are confined. In the space between the

157 subdomains, EGFR molecules are allowed to diffuse in the fast-mobile state. The

158 cholesterol depletion loosens the confinement and enlarges the range of slow-mobile

159 motion (Fig. 2c, left middle), possibly leading to occasional subdomain fusion. In the

160 case of sphingomyelin-depletion,slight expansion of the diffusion area was observed

161 (Fig. 2c, left bottom), reflecting the increase in both the confinement length of the slow-

162 mobile state and the diffusion coefficient of the fast-mobile state.

\section{Fig. 2}
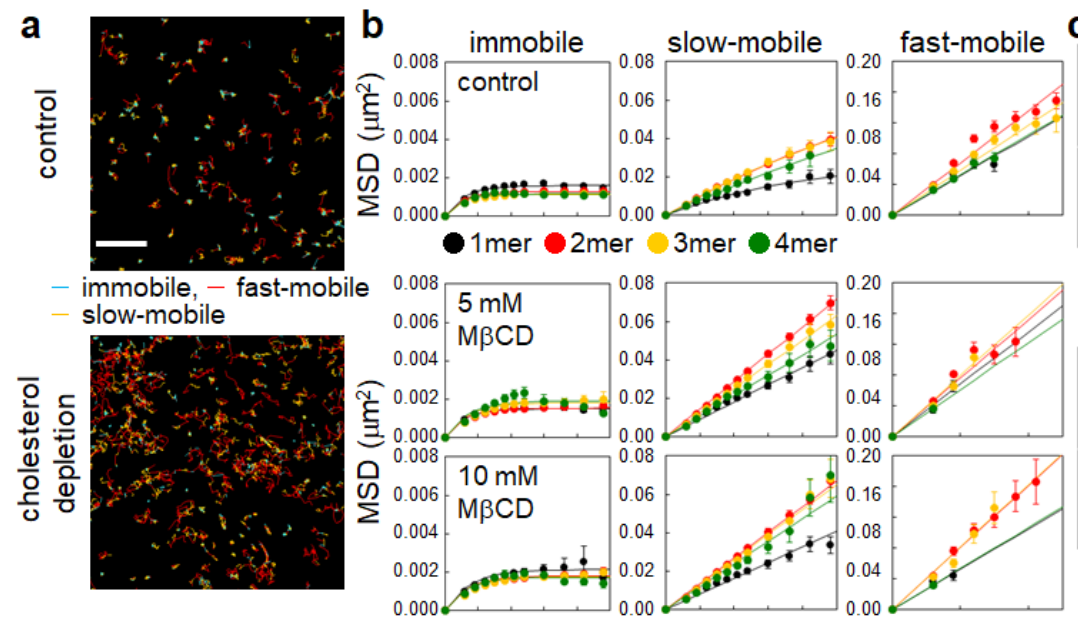

C
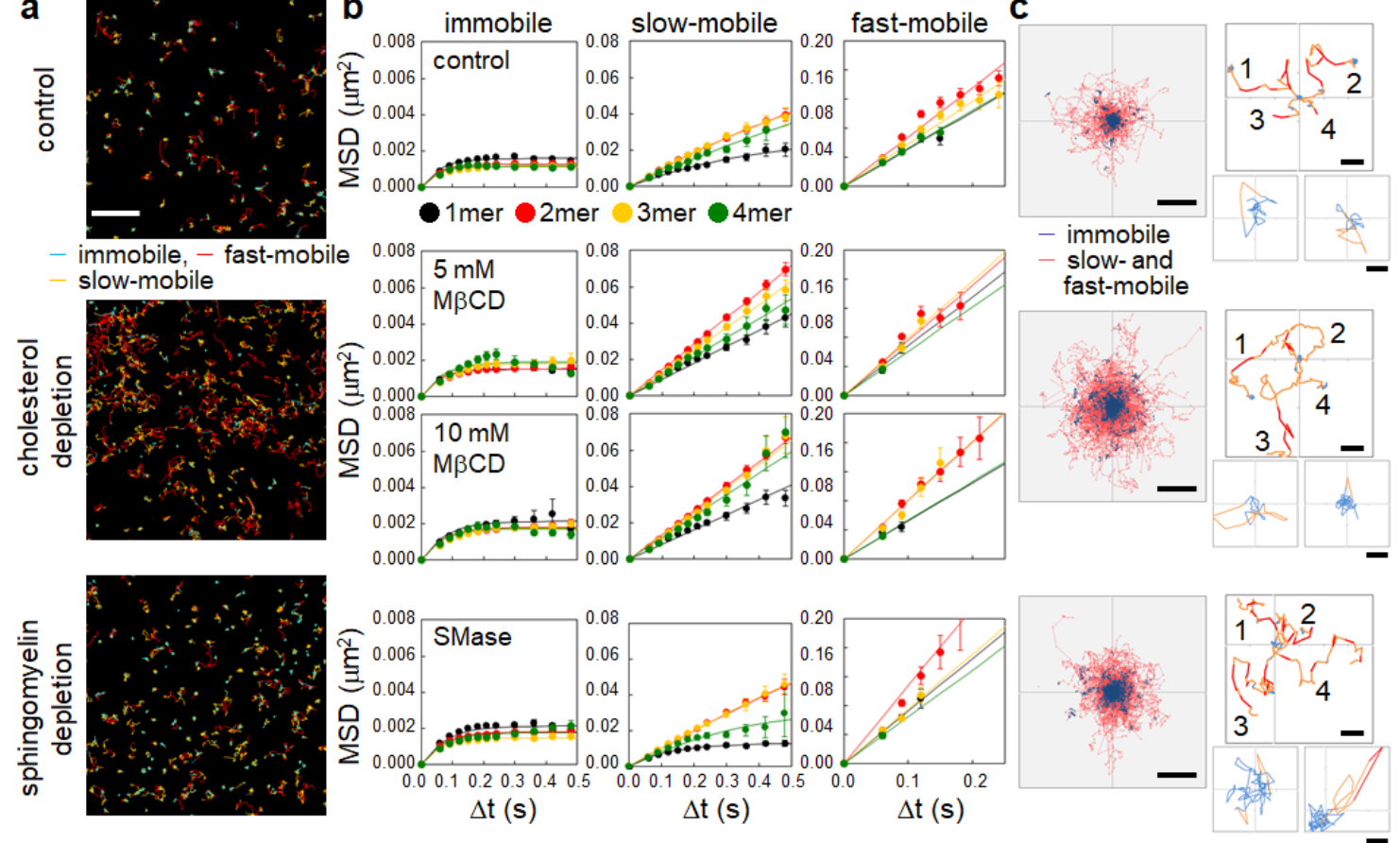
164 Lipid-depletion and EGFR mobility in the absence of EGF. a. Trajectories of EGFR

165 particles in three state transitions. Scale bar, $2 \mu \mathrm{m}$. b. MSD- $\Delta \mathrm{t}$ plots for each motional

166 and clustering state. Data are shown as the mean and SE. All single-cell data points are

167 shown in Fig. S4. c. Trajectories of 500 particles up to 50 frames $(1.5 \mathrm{sec})$ originated

168 from the immobile region were superimposed (left). Scale bar, $500 \mathrm{~nm}$. Typical

169 trajectories were extracted in the right panels. The number denotes each trajectory.

170 Scale bars, $300 \mathrm{~nm}$. In the smaller panels, trajectories that came back to the identical

171 immobile region are shown. Scale bars, $50 \mathrm{~nm}$.

173 Cholesterol depletion increased the slow-mobile EGFR pre-dimer.

174 The cluster size distribution, which was obtained from the HMM analysis, showed that

175 EGFR dimer and higher-order clusters existed even without ligand stimulation and thus

176 could be called pre-dimer and pre-clusters, respectively. The pre-dimer is responsible

177 for increasing the sensitivity of EGF signaling (Hiroshima et al., 2012; Teramura et al.,

178 2006), but spontaneous auto-phosphorylation hardly occurs. Ligand binding alters the

179 pre-dimer to an active configuration (Hofman et al., 2010), enabling auto-

180 phosphorylation. We found that when cholesterol was depleted, the fraction of pre-

181 dimer in the slow-mobile state was significantly increased (1.4-fold), but the fractions of

182 monomers and higher-order clusters ( $\geq$ trimer) were unchanged (Fig. 3a and S6),

183 suggesting an upshift in dimerization affinity between EGFR monomers and the

184 destabilization of clusters to the dimer. The fractions from monomer to tetramer were

185 reduced in the fast-mobile state, but no change was observed in the immobile state

186 (Table S2). These changes increased the total slow-mobile fraction 1.3-fold (Fig. S3b

187 and Table S1). 
We calculated the influx and efflux of monomers and pre-dimers from the immobile and fast-mobile states to the slow-mobile state based on the HMM analysis

190 (Tables S1 and S2). In the control condition, a significant net efflux of monomers $(0.27$

$191 \pm 0.05 \% \mathrm{~s}^{-1}$ ) was observed from the slow-mobile state, but there was no significant influx or efflux of the dimers. Under cholesterol-depletion, influx and efflux were

193 balanced both for monomers and dimers in the slow-mobile state, thus producing no net

194 flux. Although the decreased efflux of the monomer fraction from the slow-mobile state under cholesterol-depletion can induce pre-dimer formation, considering the $11.5 \%$

196 fraction of the slow-mobile state, this effect is small within the reaction time

197 (subsecond) of the dimerization and decomposition (see below). However, this

198 decreased efflux increased the fraction of the slow-mobile state. Because $49 \%$ and $63 \%$

199 of pre-dimer formations were observed during the slow-mobile state in the control and

200 cholesterol-depleted conditions, respectively, the increase in the slow-mobile fraction

201 increased the fraction of pre-dimers in the cholesterol-depletion condition. Transitions

202 in the motional states correlated neither with the pre-dimer formation nor the

203 decomposition.

We also measured the reaction rate constants of dimerization and dimer decomposition. The 1st-order dimerization rate constants in the slow-mobile state, were calculated from the frequency of dimerization events and found to be $7.3 \pm 0.5 \mathrm{~s}^{-1}$ (123 cells) in the control condition and $7.4 \pm 0.4 \mathrm{~s}^{-1}$ (69 cells) in the cholesterol-depleted condition. The difference was not statistically significant. The decomposition rate constants were $7.6 \pm 0.05 \mathrm{~s}^{-1}$ under the control condition and $6.1 \pm 0.06 \mathrm{~s}^{-1}$ in the

210 cholesterol-depleted condition, which was a significant difference (Table S3). The total

211 density of fluorescent particles on the cell surface $\left(1.4 \pm 0.7 \mu \mathrm{m}^{-2}\right)$ was not affected by

212 the cholesterol depletion. When this particle density was applied to the region for slow-

213 mobile motion, the fractions of monomers and dimers in the slow-mobile state were converted into particle densities of $0.32 \pm 0.02 \mu \mathrm{m}^{-2}$ and $0.67 \pm 0.04 \mu \mathrm{m}^{-2}$ in the control 
215 condition, and $0.28 \pm 0.02 \mu \mathrm{m}^{-2}$ and $0.74 \pm 0.04 \mu \mathrm{m}^{-2}$ in the cholesterol-depletion

216 condition and resulted in dissociation constants $\left(\mathrm{K}_{\mathrm{d}}\right)$ of $0.33 \pm 0.02 \mu \mathrm{m}^{-2}$ and $0.23 \pm 0.02$

$217 \mu \mathrm{m}^{-2}$, respectively. Here, the decrease in the dissociation rate constant, i.e., the increase

218 in the stability of pre-dimers, mainly contributed to the increased pre-dimer formation

219 under the cholesterol depletion. Furthermore, the destabilization of the EGFR clusters

220 suggested above may have another cause to increase the pre-dimer fraction.

Next, we checked locations of the pre-dimer formation relative to the center of the first immobile region along the single-molecule trajectories. The frequency of

223 dimerization events per area in the slow- and fast-mobile states was mapped in two dimensions and averaged over the circumference (Fig. 3b). Reflecting the release from confinement (Fig. 2b), the pre-dimerization locations spread further away when cholesterol was depleted ("M $\beta C D$ " in Fig. 3b). Most of the molecules traveled to the next immobile region (long-traveled; Fig. 3c) and formed pre-dimers regardless of the lipid condition (Fig. 3d). In the absence of EGF, the long-traveled fraction was largest upon cholesterol-depletion (Fig. 3e). Dimerization and higher-order clustering also occurred more frequently in the long-traveled fraction (Fig. 3f). These results suggest

231 that cholesterol-depletion spreads the pre-dimers and pre-clusters (Fig. S7a) of EGFR 232 over a large region of the plasma membrane.

234 regarding the EGFR clustering, such as the fraction distribution (Fig. 3a and S6),

235 reaction rate constants (Tables $\mathrm{S} 1$ and S2), location of the dimerization (Fig 3b, d, and

236 f), or fraction of long-traveled molecules (Fig. 3d). 
Fig. 3
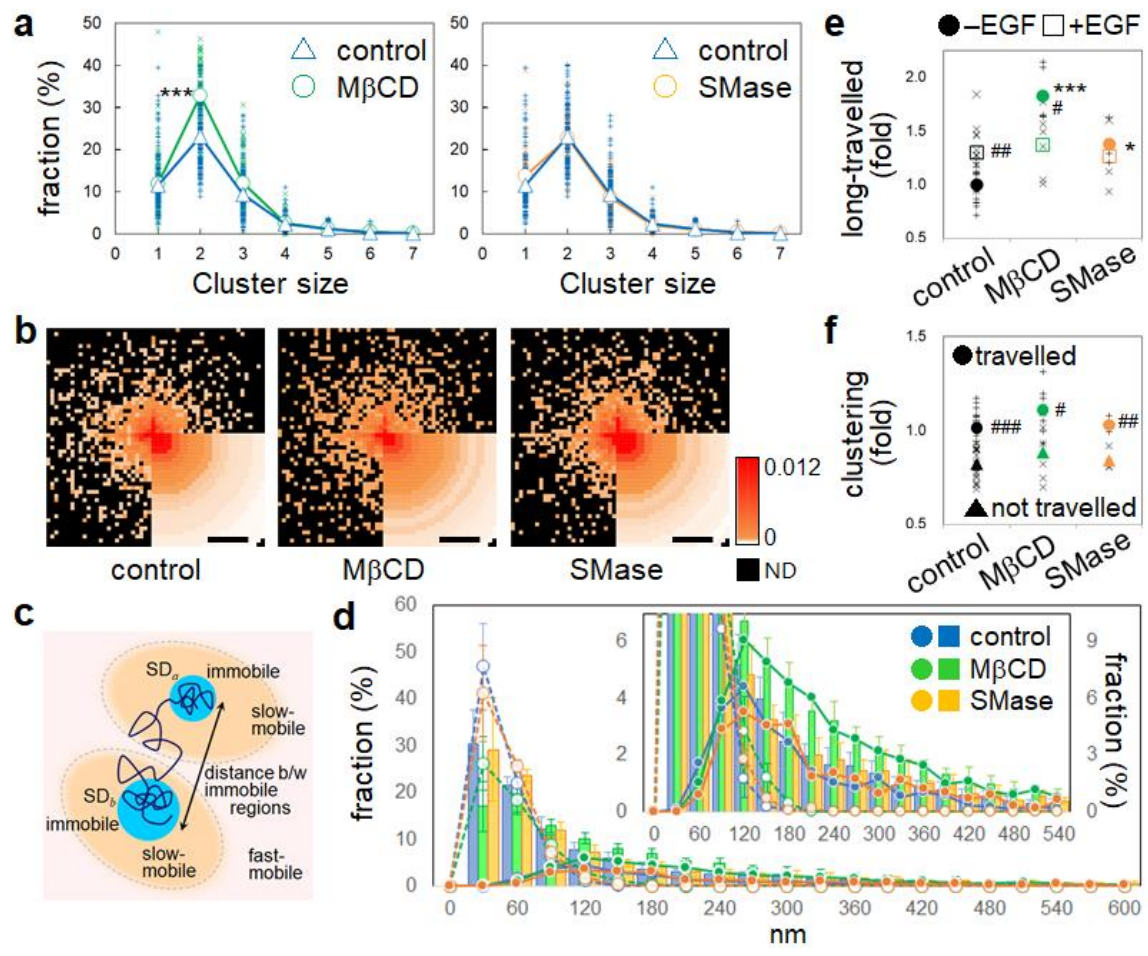

240 Lipid-depletion and EGFR pre-dimerization. a. Fractions of clusters belonging to the

241 slow-mobile state. Averages (circles and triangles) and single-cell data $(+, \times)$ are shown.

242 b. Locations of pre-dimer formation of the mobile (slow and fast) EGFR molecules

243 relative to the center of the first immobile region in each trajectory. The color code

244 indicates the number of events (trajectory ${ }^{-1} \mathrm{~s}^{-1}$ ). Scale bars, $500 \mathrm{~nm}$. c. A schematic

245 illustration of an EGFR trajectory. Movements longer than the distance between two

246 immobile regions are called "long-traveled". d. Distribution of the traveling distances

247 during $1.5 \mathrm{sec}$ (circles; left scale) and normalized frequencies of pre-dimer formation

248 (bars; right scale) during the slow- and fast-mobile states (percentage of total events).

249 Dots indicate distributions for the long-traveled EGFR molecules, and hollow circles

250 indicate other molecules. Inset: the distributions with small fractions are magnified. e.

251 Relative fractions of long-traveled EGFR moved out of an immobile region during the

252 observation period. f. Fractions of EGFR molecules with clustering ( $\geq$ dimer) in the

253 mobile state after moving out of an immobile region (-EGF). In $\mathbf{e}$ and $\mathbf{f}$, the fractions are 
normalized to control cells. $* * *$ and $* \mathrm{p}<0.001$ and $<0.05$ (t-test), respectively, between control and lipid-depleted conditions. \#\#, \#\#, and \# $\mathrm{p}<0.001,<0.01$, and $<$ 0.05 (t-test), respectively, between before and after EGF stimulation.

We quantitatively described the extent of EGF-induced clustering as the ratio of cluster

261 fractions before and after EGF stimulation (Figs. 4a and S8a) and noticed obvious effects of the lipid depletion in the immobile state. In the control condition, the ratio for the same sized clusters larger than dimers was increased up to 7.0-fold, indicating that EGF stimulation reinforced higher-order clustering. When either cholesterol or sphingomyelin was depleted, this increase was strongly inhibited (only up to 2.5-fold), indicating that lipids facilitate the EGF-induced cluster formation. At the same time, the EGF-induced expansion of the locations of clustering ( $\geq$ trimer) was observed under the control condition but not under lipid-depletion (Fig. 4b). However, the area of the EGFinduced dimerization was similar under all lipid conditions (Fig. S7b). The EGFinduced dimerization during long traveling occurred at the same frequency between all lipid conditions (Fig. 4c), whereas higher-order clustering was facilitated by EGF only

272 in the control condition (Fig. 4d) in parallel with the increase in long-traveled molecules 273 (Fig. 3e). molecules were immobilized at the same time as higher-order clustering in the control condition. We previously reported that EGF stimulation of cells transiently releases the confinement of the slow-mobile diffusion at the very early stage ( $30 \mathrm{sec})$, then shrinks 
279 were formed during this biphasic mobility change (Hiroshima et al., 2018), which

280 depends on the EGF concentration (Yasui et al., 2018). Our observations in the present

281 study confirmed that EGF induces clustering of long-traveled particles and shrinks the

282 area for the slow-mobile state under the control and sphingomyelin-depleted conditions

283 (Fig. 4e and S4) and reduced the confinement lengths to $\sim 160$ (monomer) and $\sim 340 \mathrm{~nm}$

284 ( $\geq$ dimer) and $\sim 60$ (monomer) and $\sim 530 \mathrm{~nm}$ ( $\geq$ dimer), respectively (Table S1). Even

285 for molecules in the slow-mobile state moving without confinement under cholesterol-

286 depletion, the diffusion coefficient and MSD were significantly decreased by EGF. The

287 EGF-induced reduction in EGFR mobility was independent of higher-order clustering

288 and not regulated by cholesterol or sphingomyelin.

289 Fig. 4

a

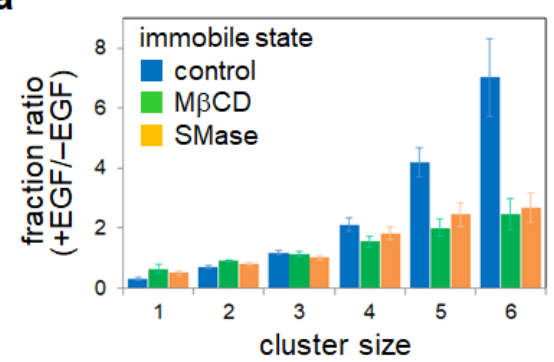

c

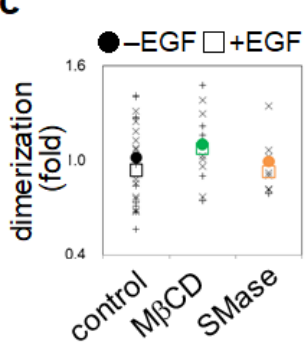

b

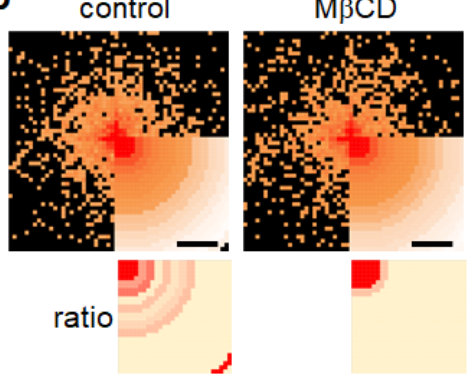

SMase

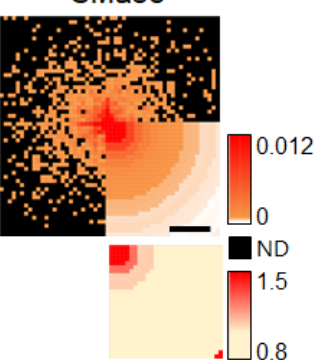

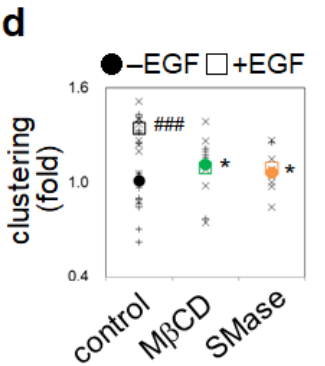

e slow-mobile

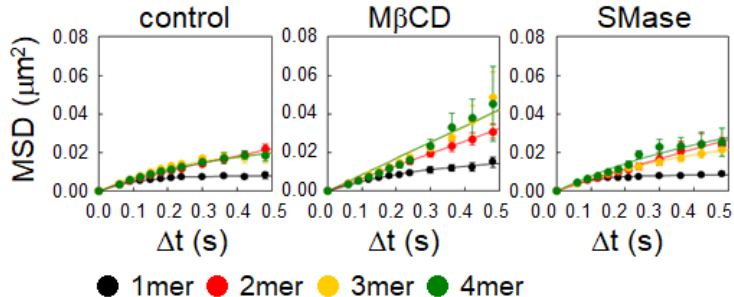

290 Lipid-depletion and EGF induced changes in mobility and clustering. a. The ratio

291 of immobile clusters before and after EGF stimulation. Means and SE from 153 and 25

292 cells for control, 69 and 21 cells for cholesterol-depletion, and 50 and 27 cells for

293 sphingomyelin-depletion are shown before and after the EGF stimulation, respectively.

294 b. Top, locations of EGF-induced higher-order clustering during the mobile state 
295 (trajectory $^{-1} \mathrm{~s}^{-1}$ ) relative to the center of the first immobile region. Bottom, ratios of 296 clustering (after:before the EGF addition). Scale bars, $500 \mathrm{~nm}$. c and d. Dimerization (c)

297 and higher-order clustering ( $\geq$ trimer; d) events as the relative fraction among the total 298 long-traveled molecules. The fractions are normalized to control cells before the EGF 299 stimulation. * $\mathrm{p}<0.05$ (t-test) between control and lipid-depleted conditions; \#\#\# $\mathrm{p}<$ $300 \quad 0.001$ (t-test) between before and after EGF stimulation. e. MSD- $\Delta t$ plots of the slow301 mobile state. Error bars: SE. All single-cell data points are shown in Fig. S4.

304 The adaptor proteins GRB2 and SHC receive signals from activated EGFR. Their SRC 305 homology 2 (SH2) domains interact with the phosphorylated tyrosine residues of EGFR

306 (Lowenstein et al., 1992), causing them to translocate to the plasma membrane

307 (Hiroshima et al., 2018; Yoshizawa et al., 2021) and result in the tyrosine

308 phosphorylation of SHC. We conducted single-molecule imaging of GRB2-HaloTag

309 labeled with tetramethylrhodamine (TMR) and a Western blotting analysis of SHC

310 (p52SHC) phosphorylation in EGFR-GFP expressing cells. The number of GRB2

311 molecules on the plasma membrane (Fig. 5a and S9a) increased after EGF stimulation

312 2.5-fold in the control condition. Under cholesterol- and sphingomyelin-depletion, the

313 increase of translocated GRB2 molecules was 1.0- and 1.5-fold, respectively. After

314 cholesterol- and sphingomyelin-depletion, the EGF-induced phosphorylation of SHC

315 Tyr 317 residue (Fig. 5b and S9b) was decreased to $0.73 \pm 0.07$ - and $0.78 \pm 0.08$-fold,

316 respectively, of the control condition (Fig. 5c). These reductions of EGFR/GRB2 and

317 EGFR/SHC interactions by cholesterol-depletion were in contrast to the upregulated

318 EGFR phosphorylation (Fig. 1). 
319 Cholesterol- or sphingomyelin-depletion inhibited ERK and AKT

320 phosphorylation.

321 The phosphorylation of the downstream protein ERK in the EGFR signaling was

322 measured by time-course Western blotting and maximized 5 minutes after the EGF

323 stimulation, which is later than the time of EGFR phosphorylation (Figs. 5d and S10a).

324 EGF-induced ERK phosphorylation was observed in all conditions, but its level was

325 lower with cholesterol-depletion, which is expected when considering the reduced

326 GRB2 translocation to the plasma membrane (Fig. 5a) and SHC phosphorylation (Fig.

$3275 \mathrm{~b}$ and $\mathrm{c}$ ). Unlike the adaptor proteins, the reduction of EGF-induced phosphorylation

328 was remarkable in ERK under cholesterol-depletion. The dependency on EGF

329 concentration was quantified for the phosphorylation of ERK and AKT, another

330 downstream protein, 2 minutes after the stimulation (Figs. 5e and S10b). The $\mathrm{EC}_{50}$

331 values for the control and cholesterol-depleted conditions, which were obtained by

332 fitting the dose-response curve (see Methods), were almost the same (within $\pm 1 \mathrm{nM}$ ).

333 The effects of the lipid depletions on the phosphorylation of AKT and ERK were

334 evaluated 2 minutes after $30 \mathrm{nM}$ EGF stimulation, which is almost the saturation

335 condition (Figs. 5f and S10b). Although cholesterol-depletion increased the level of

336 EGFR phosphorylation (Fig. 1e), the levels of AKT and ERK phosphorylation were

337 significantly decreased (Fig. 5f). Sphingomyelin-depletion also lowered the levels of

338 ERK and AKT phosphorylation, but differently, likely reflecting the specificities of the

339 signaling pathways. 


\section{Fig. 5}

a
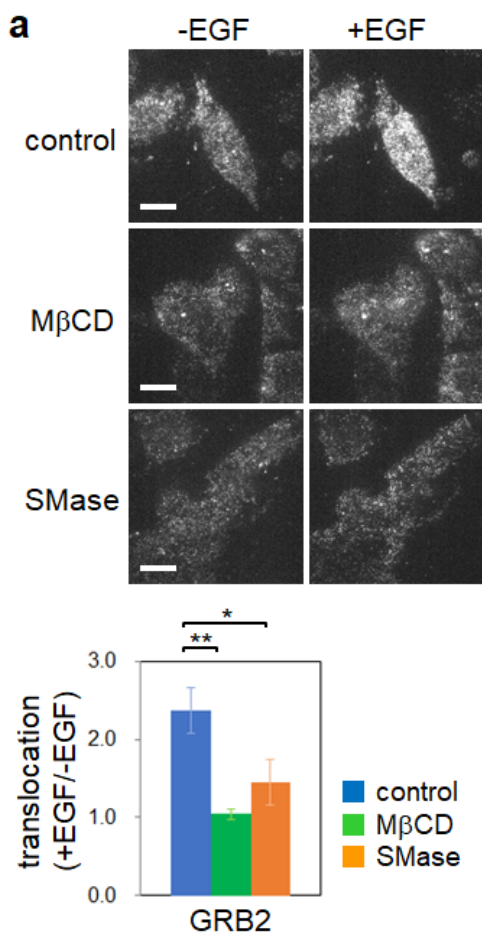

b

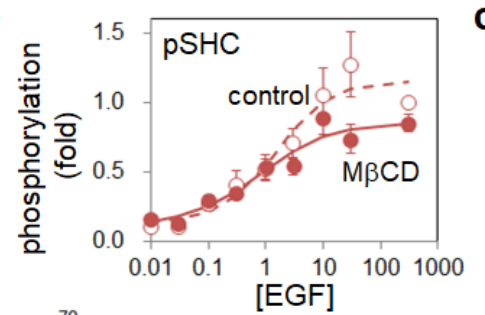

\section{d}

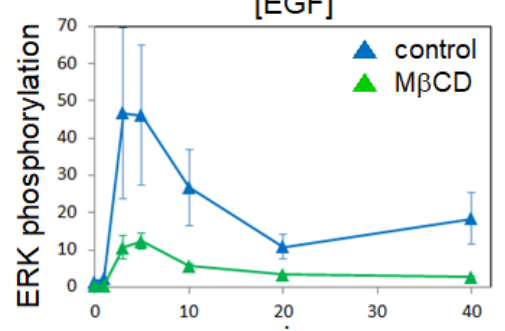

e

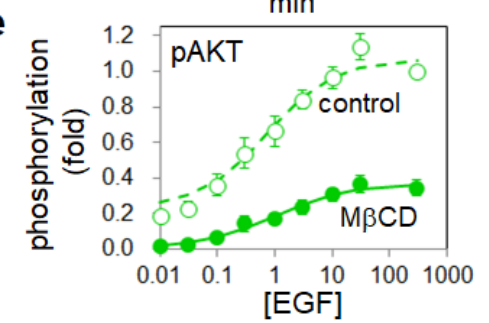

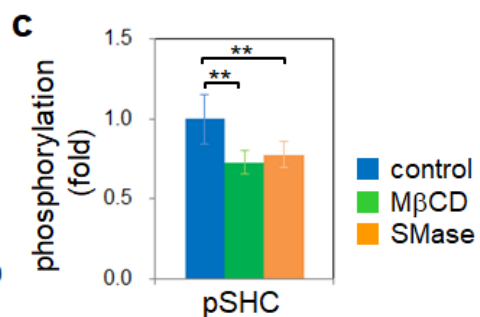
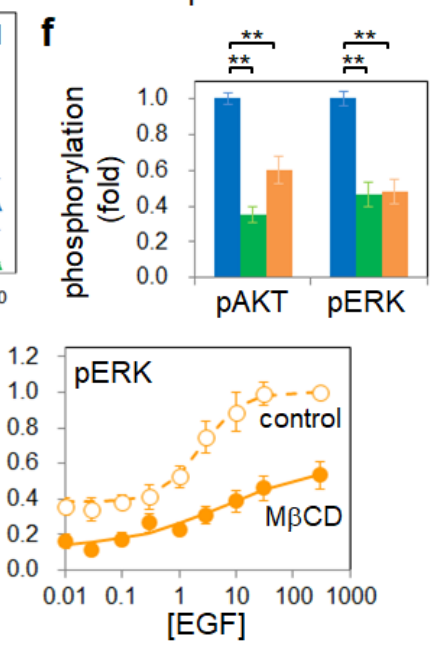

344 Lipid-depletion and downstream signaling. a. Translocation of GRB2. Top, single-

345 molecule images of GRB2-HaloTag::TMR on the plasma membrane. Scale bars: $10 \mu \mathrm{m}$.

346 Bottom, the ratio of single-molecule brightness on the plasma membrane in the same

347 cells before and after the EGF stimulation (average of 6-8 trials). b. Dose-response

348 curves for the EGF-induced phosphorylation of SHC Y317 (average of 6 trials). c.

349 Phosphorylation levels of SHC at 30 nM EGF. d. Time-course of the EGF-induced

350 ERK phosphorylation (average of 3 trials). Fold-changes of the phosphorylation level

351 relative to 0 min. e. Dose-response curves for EGF-induced phosphorylation of

352 downstream proteins (average of 7 and 13 trials for AKT and ERK, respectively). f.

353 EGF-induced phosphorylation levels of AKT and ERK. * and ** $\mathrm{p}<0.05$ and $\mathrm{p}<0.01$,

354 respectively. Error bars: SE. All data points are shown in Fig. S2b, S8, S9b, and S9c. In

$355 \mathbf{a}, \mathbf{b}$, and $\mathbf{e}$, the phosphorylation was measured 2 min after 30 nM EGF stimulation. 


\section{Discussion}

358 Cholesterol and sphingomyelin are major components of the plasma membrane

359 subdomain where EGFR has been known to accumulate. We investigated how the

360 depletion of either component affects EGFR behavior and signaling. We confirmed that

361 the depletion of one lipid on the plasma membrane does not affect the other (Fig. S1)

362 (Abe et al., 2012). Therefore, the observed phenomena in the present study, including

363 downstream signaling of EGFR, were assumed to reflect the effects of each lipid.

EGFR phosphorylation in the early stage of EGF stimulation was upregulated under cholesterol-depletion (Figs. 1d and e). We considered this upregulation to depend on the increased amount of EGFR pre-dimer, which hardly undergoes auto-

367 phosphorylation but is primed for a rapid response upon EGF stimulation (Teramura et al., 2006). EGFR has three motional modes in its lateral diffusion coefficient. After cholesterol-depletion, the amount of pre-dimer increased approximately 1.4 -fold

370 primarily in the slow-mobile state. The diffusion mode of the slow-mobile state was 371 altered from confined to simple diffusion (Fig. 2b) without a significant change in the

372 diffusion coefficient (Fig. S3a). This observation indicates that cholesterol-depletion 373 enabled molecules to go freely through some barrier and move long distance $(\sim 1.8$ fold 374 longer than the control during the observation time). This barrier might correspond to a

375 physical factor that maintains spatial phase separation in the membrane to impede

376 EGFR from moving over the subdomain border composed of cholesterol or some component interacting with cholesterol around EGFR (e.g. shell model) (Anderson,

378 2002). Our results suggest that EGFR molecules in the slow-mobile state prefer to exist

379 in the subdomains. Since EGFR pre-dimers were mainly present in the slow-mobile

380 state, the disappearance of the barrier allowed them to spread over the cell surface. 
383 decomposition. In the slow-mobile state, the rate constant of decomposition was

384 significantly decreased, but we did not detect a significant change in the dimerization

385 rate constant. As a result, the affinity was increased by the cholesterol depletion. In

386 addition, the fraction of the slow-mobile state was increased due to increased and

387 decreased of the transition probabilities (rate constants) from the fast- to slow-mobile

388 states and the slow- to fast-mobile states, respectively (Table S1). These two effects

389 induced the increase in the number of slow-mobile pre-dimers under the cholesterol-

390 depleted condition and possibly resulted in the upregulation of EGFR phosphorylation.

391 The disappearance of the diffusion barrier for the slow-mobile state of EGFR molecules

392 may be related to the increase of the slow-mobile fraction. It also seems likely that the

393 stimulative effect of cholesterol-depletion on the EGFR phosphorylation (Fig. 1) was

394 caused by the stabilization of a pre-dimer structure for the kinase activation. Recently,

395 we found that a transmembrane (TM)-juxtamembrane (JM) peptide of EGFR forms

396 distinct structures of dimers in nanodiscs with or without cholesterol (Maeda et al.,

397 2021). Cholesterol suppressed the formation of the JM dimer, which can be attributed to

398 the structure suggested for EGFR kinase activation (Arkhipov et al., 2013). On the other

399 hand, cholesterol stabilized dimers and trimers of EGFR peptides with lower JM

400 interactions in the nanodiscs (Maeda et al. 2021).

401 Sphingomyelin-depletion, which did not affect cholesterol, also caused

402 significant effects on EGFR in the slow-mobile state. The confinement length for the

403 slow-mobile state was increased, though the confinement did not disappear (Fig. 2b).

404 The fraction of the slow-mobile state was increased (Fig. S3b), reflecting the rise in the

405 transition probability from the fast- to slow-mobile states (Table S1). The fractions of

406 the monomer and other clusters in the slow-mobile state were unchanged.

407 Sphingomyelin-depletion is likely to disrupt $\mathrm{PIP}_{2}$ domains, which locate at the

408 cytoplasmic side of the sphingomyelin domains on the extracellular side of the plasma

409 membrane (Abe et al., 2012). $\mathrm{PIP}_{2}$ facilitates dimer formation of the JM region of 
410 EGFR (Arkhipov et al., 2013; Maeda et al., 2018), and disruption of the PIP $_{2}$ domain

411 can cause EGFR monomerization. This disruption may be the reason for the unchanged

412 pre-dimer fraction despite the increase in the slow-mobile state.

EGFR clusters larger than dimers were also formed before the EGF stimulation

414 (pre-clusters). Different from pre-dimers, cholesterol-depletion did not increase the pre-

415 cluster fraction (Fig. 3a), although the confinement disappeared (Fig. 2b) to enlarge the

416 regions of the slow-mobile motions for the pre-clusters (Fig. 2c). On the contrary, the

417 EGF-induced formation of higher-order clusters, which was observed in the control

418 condition, was suppressed under cholesterol-depletion (Fig. 4a and 4b) in a cholesterol

419 dose-dependent manner (Fig. S8b). Sphingomyelin-depletion also suppressed the EGF-

420 induced clustering of EGFR. These lipid dependencies suggest that the clustering of

421 EGFR is caused by a mechanism different from that for EGF-induced dimerization.

422 Cholesterol and sphingomyelin may pack and enclose the EGFR molecules in small

423 membrane subdomains or directly bind up the molecules. The oligomerization of TM

424 peptides of EGFR has been observed in liposomes containing cholesterol (Jones et al.,

425 1998). Following our previous report that the EGF-induced EGFR clusters in the

426 immobile state are the primary interaction sites with the adaptor protein GRB2

427 (Hiroshima et al., 2018), the deficient clustering by the lipid depletion correlated with

428 the reduction in the membrane translocation and in the phosphorylation of adaptor

429 proteins (Fig. 5a and b). Indeed, the downstream proteins ERK and AKT showed

430 reduced phosphorylation (Fig. 5c, 5d, and 5e), suggesting that cholesterol and

431 sphingomyelin substantially contribute to the cellular signaling through the EGFR-

432 immobile cluster formation.

Based on our observations (Fig. S11), we provide a scheme for EGFR-mediated

434 cell signaling (Fig. 6): First, the immobile and slow-mobile states of EGFR are confined

435 within a cholesterol- and sphingomyelin-enriched membrane subdomain (Fig. 2b). A 
436 significant fraction of EGFR molecules forms pre-dimers while moving within and

437 between the slow-mobile state; however, only cholesterol and not sphingomyelin

438 prevents the slow-mobile EGFR from freely passing over the border and interfering

439 with the pre-dimer formation (Fig. 3a). Then, EGF association quickly converts EGFR

440 from a pre-dimer to kinase active dimer. Moreover, EGF facilitates the formation of

441 clusters larger than dimers with an enlarged area of the clustering (Fig. 4b and squares

442 in $4 \mathrm{~d}$ ). This process involves the transient expansion of EGFR-cluster (not monomer)

443 mobility at the very early stage of the EGF stimulation (Hiroshima et al., 2018). The

444 clustering of EGFR requires cholesterol and sphingomyelin (Fig. 4a). Tyrosine

445 phosphorylation of EGFR, a prerequisite for the clustering, leads to immobilization

446 (Yasui et al., 2018), though the immobilization does not require the clustering. Finally,

447 the immobile clusters increase and principally transduce information to the downstream

448 cell signaling.

Previous studies have reported both positive and negative effects of membrane

450 cholesterol and lipid rafts in EGFR phosphorylation and downstream signaling (Chen

and Resh, 2002; Fang et al., 2006; Liu et al., 2007; Zhuang et al., 2002). Here, we

452 observed the dimerization and clustering of EGFR at single-molecule resolution and

453 found a dichotomic effect of cholesterol, in which cholesterol suppresses the pre-

454 dimerization of EGFR, leading to a reduction of EGF-induced phosphorylation, but

455 assists with the EGF-induced higher-order clustering of phosphorylated EGFR to

456 construct reaction sites for downstream signaling. This latter effect is common with

457 sphingomyelin. The molecular mobility, dimerization/clustering, phosphorylation, and

458 interaction with downstream molecules are intricately coupled in the process of EGFR

459 signaling. Changes in the receptor behavior and membrane lipid environment can

460 therefore cause variable results in the signal transduction, potentially causing the EGFR

461 related diseases such as cell carcinomas, dyslipidemia, and so forth. 
462 Fig. 6
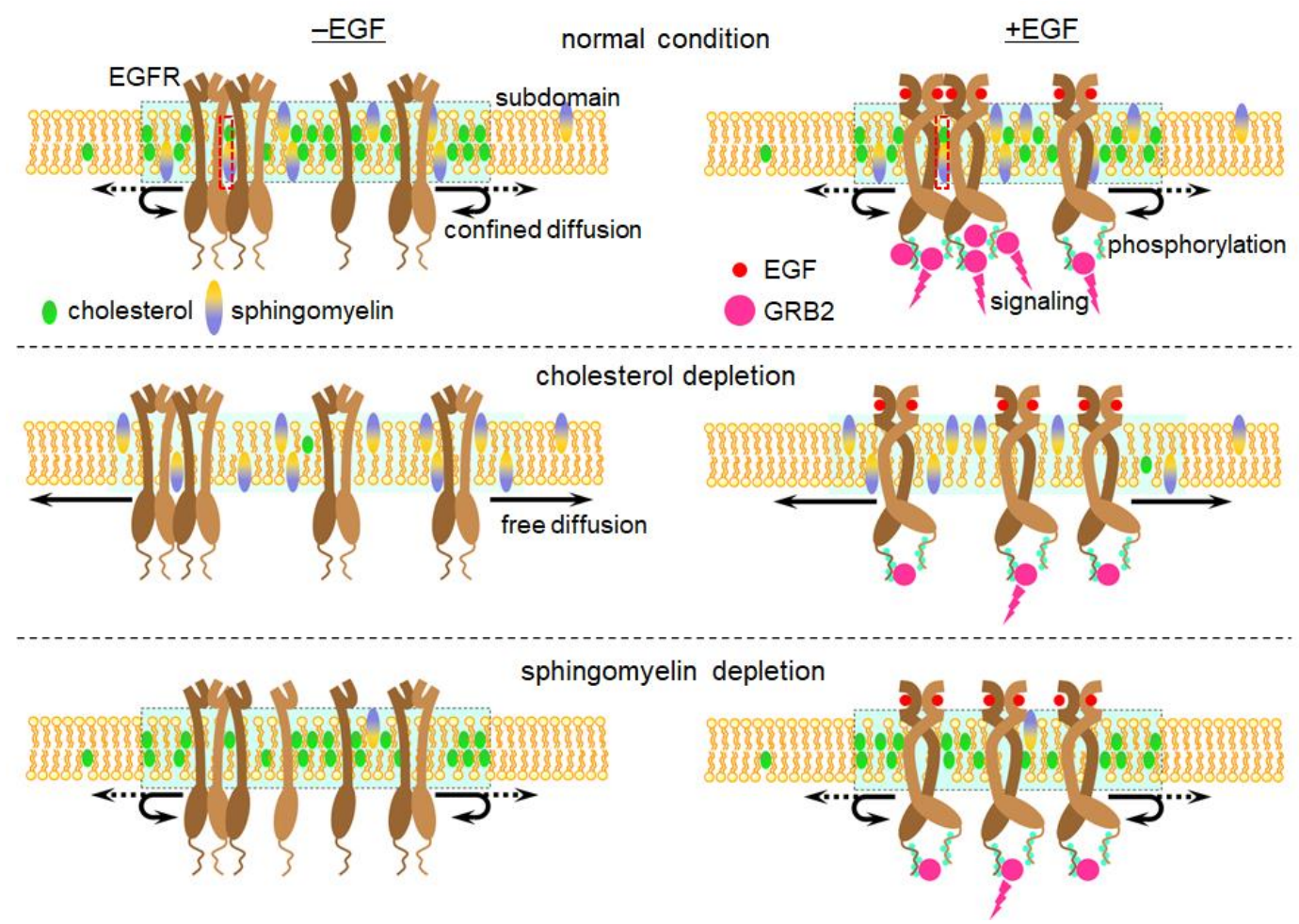

463 Lipid-depletion and EGFR signaling. Proposed EGFR dynamics in the slow-mobile

464 state under control and lipid-depleted conditions. Dashed blue rectangles indicate the

465 membrane subdomains that confine the EGFR mobility. Molecules clustered and

466 immobilized during the slow-mobile state relay EGF-induced signaling depending on

467 the lipid components. 


\section{Acknowledgment}

469 We thank A. Yoshimura for the cDNA, H. Sato and A. Kanayama for experimental

470 support, and P. Karagiannis for reading the manuscript. This study is supported by

471 MEXT Japan with Grants-in-Aid for Scientific Research(B) (18H01839) and Grant-in-

472 Aid for Scientific Research on Innovative Areas (18H05414). Y.S. was supported by

473 MEXT Japan with Grants-in-Aid for Scientific Research (19H05647) and JST with

474 CREST (JPMJCR1912).

\section{Author Contributions}

477 M.H. and Y.S. designed the research; M.H. performed the experiments and analyzed the

478 data; M.A. purified the fluorescence probes for lipids; M.A. and A.M. directed the lipid

479 depletion study; N.T. and F. H-M quantified cellular cholesterol; M.U. and T.K.

480 directed the study; and M.H. and Y.S. wrote the paper.

\section{Declaration of Interests}

483 The authors declare no competing financial or non-financial interests. 


\section{Materials and Methods}

\section{Gene Construction}

486 The EGFR-GFP plasmid was constructed using the cDNA of human EGFR

487 (pNeoSR $\alpha I I)$ provided by Akihiko Yoshimura (Keio University) and was inserted into

488 the pEGFP-C1 vector (Clontech) with the same linker sequence suggested by Carter and

489 Sorkin (Carter and Sorkin, 1998). The GFP sequence included the monomeric mutation

490 of A206K in the enhanced GFP (EGFP) sequence. GRB2-HaloTag was constructed

491 using the human GRB2-encoding fragment and inserted into the Halo7-C2 vector in

492 which the monomeric EGFP sequence in the pEGFP-C2 vector (Clontech) was

493 substituted to the Halo7 sequence from the FN19K HaloTag T7 SP6 Flexi Vector

494 (Promega).

\section{Cell Culture and Transfection}

496 Chinese hamster ovary K1 (CHO-K1) cells were provided by RIKEN BRC through the

497 National Bio-Resource Project (MEXT, Japan). For single-molecule and Western

498 blotting experiments, a CHO cell line expressing EGFR-GFP was established. HAM

499 F12 medium supplemented with $10 \%$ fetal bovine serum (FBS) was used to maintain

500 the cells at $37{ }^{\circ} \mathrm{C}$ under $5 \% \mathrm{CO}_{2}$.

\section{Cholesterol and sphingomyelin depletion}

502 To deplete cholesterol, the cells were incubated in 5 or $10 \mathrm{mM} \mathrm{M} \beta \mathrm{CD}$ (Sigma C4555)

503 in Hank's balanced salt solution (HBSS) for 1 hour at $37^{\circ} \mathrm{C}$ under $5 \% \mathrm{CO}_{2}$. Free

504 cholesterol in the cells was separated by thin-layer chromatography (TLC) and

505 quantified using gas chromatography-flame ionization detector (GC-FID, Shimadzu

506 GC-14AH) or -mass spectrometry (GC/MS, JEOL JMS-700V). Cholesterol extent was 
507 also observed under a fluorescence microscope (Nikon, Ti) with a 20X objective lens

508 (Nikon VC 20X, NA0.75) in $\theta$ toxin-GFP labeled cells. Sphingomyelin was depleted by

509 incubating the cells in HBSS containing 1:300 diluted sphingomyelinase (Sigma,

510 S9396) for 1 hour at $37{ }^{\circ} \mathrm{C}$ under $5 \% \mathrm{CO}_{2}$. The depletion was confirmed by

511 fluorescence microscopy in lysenin-GFP labeled cells. The average fluorescence

512 intensity (per pixel) was measured over the cell region, and the averaged background

513 intensity acquired from regions with no cells was subtracted from the signal.

\section{Microscopy and Image Analysis for Single-molecule Imaging and Tracking}

515 Cell starvation was carried out by changing HAM F12 medium to modified Eagle's

516 medium minus phenol red and FBS 1 day before single-molecule imaging. Objective-

517 type total internal reflection illumination was applied to observe EGFR-GFP in the basal

518 plasma membrane of the cells through a PlanApo 60× NA 1.49 objective (Nikon,

519 Tokyo, Japan) equipped on an inverted microscope (TE2000; Nikon). Lasers with

520 wavelengths of $488 \mathrm{~nm}$ (Sapphire 488; Coherent, Santa Clara, CA) and $561 \mathrm{~nm}$

521 (Sapphire 561; Coherent) were used for the excitation of GFP and TMR, respectively.

522 The dichroic mirror and emission filter were Di02-R488 (Semrock) and FF01-525/45

523 (Semrock) for GFP, and Di02-R561 (Semrock) and BLP02-561R (Semrock) for TMR

524 imaging. An electron-multiplying CCD (EMCCD) camera (C9100-23; Hamamatsu,

525 Hamamatsu, Japan), which was controlled using HCImage software, acquired

526 fluorescence images at a frame rate of $33 \mathrm{~s}^{-1}$. The imaging was done at $25^{\circ} \mathrm{C}$. Image

527 processing was carried out with moving averages over two frames and background

528 subtraction using rolling ball filtering (radius: 25 pixels) of the ImageJ plugins. Single-

529 molecule tracking was performed on the processed images with custom-made software.

530 The obtained data including positions and intensities of all fluorescent spots were

531 analyzed using the methods described below. 
532 State Estimation Using a Hidden Markov Model with the Variational Bayes (VB533 HMM) Method

534 A time series of step displacements and fluorescence intensities of the EGFR-GFP spots

535 in the single-molecule tracking data were analyzed by VB-HMM analysis. This analysis

536 consisted of the following steps (details are given in Okamoto and Sako, 2012; Persson

537 et al., 2013). First, the data were grouped into $N$ number of states with the K-means

538 clustering method. Second, the initial parameters were calculated for each group based

539 on observation probability models describing a two-dimensional diffusion equation for

540 the step displacement and a Gaussian function for the fluorescence intensity. Third, the

541 posterior probability distribution, $q(Z, \theta)$, where $Z$ is the molecular state sequence and $\theta$

$542=\{\pi, A, \phi\}$ is the parameters of the initial values, transition matrix, and the observation

543 probability, respectively, was factorized as $q(Z) q(\theta)$. Then, the distribution functions,

$544 q(Z)$ and $q(\theta)$, were optimized with the VB expectation-maximization (VB-EM)

545 algorithm. The VB-E and VB-M steps were alternately applied to optimize $q(Z)$ with the

546 forward-backward algorithm (Bishop, 2006) and $q(\theta)$ by updating the parameters,

547 which were used in the next VB-E step. Fourth, the lower bound of the evidence, $L_{q}$,

548 was calculated to evaluate its convergence (except for the first $L_{q}$ value) by judging

549 whether the difference from the previous $L_{q}$ was less than $0.001 \%$. Fifth, if $L_{q}$ was not

550 convergent, the next iteration was performed by repeating the third and fourth steps.

551 Finally, the state sequence was determined by choosing the state with the highest

552 probability at every frame.

554 The MSD of a specific mobility and clustering state, which was attributed to steps along

555 the receptor trajectory, was calculated as

$556 M S D(n \cdot \delta t)=\left\{[x(n \cdot \delta t)-x(0)]^{2}+[y(n \cdot \delta t)-y(0)]^{2}\right\}$, where $n$ represents the frame 
557 number, $x$ and $y$ the particle positions, $\delta t$ the time interval between frames (30 ms), and

558 [ ] the average over the particles. By comparing the goodness of fit to equations for

559 confined or simple diffusion using AIC (Eq. 4), the suitable diffusion model was

560 determined for an MSD plot. The MSD calculation and all statistical tests were

561 performed using Microsoft Excel.

\section{Translocation assay of adaptor proteins}

563 Cell lines expressing both EGFR-GFP and GRB2-HaloTag were incubated in 96-well

564 plates and starved 1 day before the experiment. The HaloTag-fused adaptor protein was

565 labeled with 1-4 nM (depending on the GRB2 expression level) TMR and observed

566 with 561-nm laser light for the excitation. For large-scale single-molecule analysis with

567 high efficiency, well plate-based measurements were performed with the automated

568 system that we developed (Yasui et al., 2018). Each of the automatically determined 5

569 fields of view, including 1-3 cells per field, was observed for 200 frames $(6 \mathrm{sec})$ both

570 before and 2 minutes after the EGF stimulation. The acquired images were analyzed

571 using built-in software for tracking fluorescent spots. The spots observed in the 10th

572 frame were used for the analysis to exclude fluorescence debris, which was bleached

573 immediately after illumination. The number of translocated proteins on the plasma

574 membrane was reflected in the total brightness of the fluorescent spots, in which more

575 than one adaptor protein molecule might be included in a spot. The total brightness

576 before and after the EGF stimulation were compared by their ratio.

\section{Western blot assay}

578 The phosphorylation of proteins was quantified by western blotting using antibodies

579 against pEGFR (\#4407 for pY1173 and \#3777 for pY1068; Cell Signaling Technology

580 (CST)), pSHC (CST \#2431 for pY317), pERK (CST \#9106), and pAKT (CST \#4060) 
581 to detect tyrosine or serine/threonine phosphorylation, and antibodies against EGFR

582 (SC-03; Santacruz), SHC (CST \#2432), ERK (CST \#4696), and AKT (CST \#9272) to

583 detect protein expressions. Antibody binding was detected by luminescence using

584 1:2000 diluted HRP-linked anti-IgG antibodies (CST \#7074 for rabbit and CST \#7076

585 for mouse) as the secondary antibodies and ECL prime reagent (GE Healthcare). The

586 luminescence intensities were measured using ImageJ software (NIH). Rectangular

587 regions of interest were set in the signal (band) and background (far enough from the

588 signal) regions. The difference in the average intensities of the two regions was defined

589 as the band intensity. For the time-course analysis, the fold-change of the

590 phosphorylation level at 0 min was calculated. The obtained band intensity at each time

591 point in all conditions was normalized to that at $1 \mathrm{~min}$ of the control cells measured in

592 the identical experiment. The intensity at 0 min was significantly weak, coupled with a

593 relatively high level of noise, and not suitable as a normalization factor. Next, the

594 normalized values at $0 \mathrm{~min}$ in all conditions were averaged and used as the denominator

595 for the values at each time point. For the dose-response analysis, the obtained band

596 intensity at each EGF concentration in all conditions was normalized to that at $300 \mathrm{nM}$

597 EGF of the control cells measured in the identical experiment. The dose-response curve

598 was fitted with the Hill equation as follows:

599

$$
\text { phosphorylation }=\text { bottom }+\frac{\text { top }- \text { bottom }}{1+\left(\frac{E C_{50}}{[E G F]}\right)^{n}} .
$$

600 Here, $n$, top, and bottom are the fitted parameters indicating the Hill coefficient and

601 upper and lower bounds, respectively. $[E G F]$ is the concentration of EGF. 


\section{References}

603 Abe, M., Makino, A., Hullin-Matsuda, F., Kamijo, K., Ohno-Iwashita, Y., Hanada, K.,

604 Mizuno, H., Miyawaki, A., and Kobayashi, T. (2012). A role for sphingomyelin-rich

605 lipid domains in the accumulation of phosphatidylinositol-4,5-bisphosphate to the

606 cleavage furrow during cytokinesis. Mol. Cell. Biol. 32, 1396-1407.

607 Anderson, R.G.W. (2002). A Role for Lipid Shells in Targeting Proteins to Caveolae,

608 Rafts, and Other Lipid Domains. Science (80-. ). 296, 1821-1825.

609 Arkhipov, A., Shan, Y., Das, R., Endres, N.F., Eastwood, M.P., Wemmer, D.E.,

610 Kuriyan, J., and Shaw, D.E. (2013). Architecture and membrane interactions of the EGF

611 receptor. Cell 152, 557-569.

612 Carpenter, G., King, L., and Cohen, S. (1978). Epidermal growth factor stimulates

613 phosphorylation in membrane preparations in vitro. Nature 276, 409-410.

614 Carter, R.E., and Sorkin, A. (1998). Endocytosis of functional epidermal growth factor

615 receptor-green fluorescent protein chimera. J. Biol. Chem. 273, 35000-35007.

616 Chen, X., and Resh, M.D. (2002). Cholesterol depletion from the plasma membrane

617 triggers ligand-independent activation of the epidermal growth factor receptor. J. Biol.

618 Chem. 277, 49631-49637.

619 Chung, I., Akita, R., Vandlen, R., Toomre, D., Schlessinger, J., and Mellman, I. (2010).

620 Spatial control of EGF receptor activation by reversible dimerization on living cells.

$621 \quad$ Nature 464, 783-787. 
622 Cremesti, A.E., Goni, F.M., and Kolesnick, R. (2002). Role of sphingomyelinase and

623 ceramide in modulating rafts: Do biophysical properties determine biologic outcome?

624 FEBS Lett. 531, 47-53.

625 Fang, Y., Ferrie, A.M., and Li, G. (2006). Cellular functions of cholesterol probed with

626 optical biosensors. Biochim. Biophys. Acta - Mol. Cell Res. 1763, 254-261.

627 Hannun, Y.A., and Obeid, L.M. (2008). Principles of bioactive lipid signalling: Lessons

628 from sphingolipids. Nat. Rev. Mol. Cell Biol. 9, 139-150.

629 Hiroshima, M., Saeki, Y., Okada-Hatakeyama, M., and Sako, Y. (2012). Dynamically

630 varying interactions between heregulin and ErbB proteins detected by single-molecule

631 analysis in living cells. Proc. Natl. Acad. Sci. U. S. A. 109, 13984-13989.

632 Hiroshima, M., Pack, C. gi, Kaizu, K., Takahashi, K., Ueda, M., and Sako, Y. (2018).

633 Transient Acceleration of Epidermal Growth Factor Receptor Dynamics Produces

634 Higher-Order Signaling Clusters. J. Mol. Biol.

635 Hofman, E.G., Bader, A.N., Voortman, J., van den Heuvel, D.J., Sigismund, S.,

636 Verkleij, A.J., Gerritsen, H.C., and van Bergen en Henegouwen, P.M.P. (2010). Ligand-

637 induced EGF receptor oligomerization is kinase-dependent and enhances

638 internalization. J. Biol. Chem. 285, 39481-39489.

639 Jones, D.H., Barber, K.R., and Grant, C.W.M. (1998). The EGF Receptor

640 Transmembrane Domain: 2 H NMR Study of Peptide Phosphorylation Effects in a

641 Bilayer Environment $\uparrow$. Biochemistry 37, 7504-7508. 
642 Lajoie, P., Partridge, E.A., Guay, G., Goetz, J.G., Pawling, J., Lagana, A., Bharat, J.,

643 Dennis, J.W., and Nabi, I.R. (2007). Plasma membrane domain organization regulates

644 EGFR signaling in tumor cells. J. Cell Biol. 179, 341-356.

645 Lemmon, M.A., and Schlessinger, J. (2010). Cell signaling by receptor tyrosine kinases.

646 Cell 141, 1117-1134.

647 Lin, C.Y., Huang, J.Y., and Lo, L.W. (2016). Exploring in vivo cholesterol-mediated

648 interactions between activated EGF receptors in plasma membrane with single-molecule 649 optical tracking. BMC Biophys. 9, 1-11.

650 Liu, Y., Sun, R., Wan, W., Wang, J., Oppenheim, J.J., Chen, L., and Zhang, N. (2007).

651 The involvement of lipid rafts in epidermal growth factor-induced chemotaxis of breast 652 cancer cells. Mol. Membr. Biol. 24, 91-101.

653 Low-Nam, S.T., Lidke, K. a, Cutler, P.J., Roovers, R.C., van Bergen en Henegouwen,

654 P.M.P., Wilson, B.S., and Lidke, D.S. (2011). ErbB1 dimerization is promoted by

655 domain co-confinement and stabilized by ligand binding. Nat. Struct. Mol. Biol. 18,

$656 \quad 1244-1249$.

657 Lowenstein, E.J., Daly, R.J., Batzer, A.G., Li, W., Margolis, B., Lammers, R., Ullrich, 658 A., Skolnik, E.Y., Bar-Sagi, D., and Schlessinger, J. (1992). The SH2 and SH3 domain659 containing protein GRB2 links receptor tyrosine kinases to ras signaling. Cell 70, 431660442.

661 Maeda, R., Sato, T., Okamoto, K., Yanagawa, M., and Sako, Y. (2018). Lipid-Protein

662 Interplay in Dimerization of Juxtamembrane Domains of Epidermal Growth Factor

663 Receptor. Biophys. J. 114, 893-903. 
664 Maeda, R., Tamagaki-asahina, H., Sato, T., and Yanagawa, M. (2021). Threonine

665 phosphorylation regulates the molecular assembly and signaling of EGFR in

666 cooperation with membrane lipids. 1-33.

667 Makdissy, N., Haddad, K., Mouawad, C., Popa, I., Younsi, M., Valet, P., Brunaud, L.,

668 Ziegler, O., and Quilliot, D. (2015). Regulation of SREBPs by sphingomyelin in

669 adipocytes via a caveolin and Ras-ERK-MAPK-CREB signaling pathway. PLoS One

$670 \quad 10,1-33$.

671 Martin-Fernandez, M., Clarke, D.T., Tobin, M.J., Jones, S. V, and Jones, G.R. (2002).

672 Preformed oligomeric epidermal growth factor receptors undergo an ectodomain

673 structure change during signaling. Biophys. J. 82, 2415-2427.

674 Okamoto, K., and Sako, Y. (2012). Variational Bayes analysis of a photon-based hidden

675 Markov model for single-molecule FRET trajectories. Biophys. J. 103, 1315-1324.

676 Persson, F., Lindén, M., Unoson, C., and Elf, J. (2013). Extracting intracellular

677 diffusive states and transition rates from single-molecule tracking data. Nat. Methods

$678 \quad 10,265-269$.

679 Rabiner, L.R. (1989). A tutorial on hidden Markov models and selected applications in

680 speech recognition. Proc. IEEE 77, 257-286.

681 Sasset, L., Zhang, Y., Dunn, T.M., and Di Lorenzo, A. (2016). Sphingolipid De Novo

682 Biosynthesis: A Rheostat of Cardiovascular Homeostasis. Trends Endocrinol. Metab.

$68327,807-819$.

684 Semrau, S., and Schmidt, T. (2009). Membrane heterogeneity - From lipid domains to

685 curvature effects. Soft Matter 5, 3174-3186. 
686 Smith, A.J., Sugita, S., and Charlton, M.P. (2010). Cholesterol-dependent kinase

687 activity regulates transmitter release from cerebellar synapses. J. Neurosci. 30, 6116-

6886121.

689 Tabas, I. (2002). Consequences of cellular cholesterol accumulation: Basic concepts and 690 physiological implications. J. Clin. Invest. 110, 905-911.

691 Tao, R.-H., and Maruyama, I.N. (2008). All EGF(ErbB) receptors have preformed

692 homo- and heterodimeric structures in living cells. J. Cell Sci. 121, 3207-3217.

693 Teramura, Y., Ichinose, J., Takagi, H., Nishida, K., Yanagida, T., and Sako, Y. (2006).

694 Single-molecule analysis of epidermal growth factor binding on the surface of living 695 cells. EMBO J. 25, 4215-4222.

696 Valley, C.C., Arndt-Jovin, D.J., Karedla, N., Steinkamp, M.P., Chizhik, A.I., Hlavacek, 697 W.S., Wilson, B.S., Lidke, K.A., and Lidke, D.S. (2015). Enhanced dimerization drives 698 ligand-independent activity of mutant epidermal growth factor receptor in lung cancer.

699 Mol. Biol. Cell 26, 4087-4099.

700 Webb, S.E.D., Roberts, S.K., Needham, S.R., Tynan, C.J., Rolfe, D.J., Winn, M.D.,

701 Clarke, D.T., Barraclough, R., and Martin-Fernandez, M.L. (2008). Single-molecule

702 imaging and fluorescence lifetime imaging microscopy show different structures for

703 high- and low-affinity epidermal growth factor receptors in A431 cells. Biophys. J. 94, $704803-819$.

705 Yasui, M., Hiroshima, M., Kozuka, J., Sako, Y., and Ueda, M. (2018). Automated

706 single-molecule imaging in living cells. Nat. Commun. 9. 
bioRxiv preprint doi: https://doi.org/10.1101/2021.08.28.457965; this version posted August 28,2021 . The copyright holder for this preprint

(which was not certified by peer review) is the author/funder, who has granted bioRxiv a license to display the preprint in perpetuity. It is made available under aCC-BY 4.0 International license.

707 Yoshizawa, R., Umeki, N., Yamamoto, A., Murata, M., and Sako, Y. (2021). Biphasic

708 spatiotemporal regulation of GRB2 dynamics by p52SHC for transient RAS activation.

709 Biophys. Physicobiology 18, 1-12.

710 Yu, X., Sharma, K.D., Takahashi, T., Iwamoto, R., and Mekada, E. (2002). Ligand-

711 independent dimer formation of epidermal growth factor receptor (EGFR) is a step

712 separable from ligand-induced EGFR signaling. Mol. Biol. Cell 13, 2547-2557.

713 Zhuang, L., Lin, J., Lu, M.L., Solomon, K.R., and Freeman, M.R. (2002). Cholesterol-

714 rich lipid rafts mediate Akt-regulated survival in prostate cancer cells. Cancer Res. 62,

$715 \quad 2227-2231$.

716 Zidovetzki, R., and Levitan, I. (2007). Use of cyclodextrins to manipulate plasma

717 membrane cholesterol content: evidence, misconceptions and control strategies.

718 Biochim. Biophys. Acta 1768, 1311-1324. 